\title{
Bis(phthalocyaninato)yttrium Grown on Au(111): Electronic Structure of a Single Molecule and the Stability of Two- dimensional Films Investigated by Scanning Tunneling Microscopy/Spectroscopy at $4.8 \mathrm{~K}$
}

\author{
Yanfeng Zhang ${ }^{1,2}(\bowtie)$, Pengfei Guan ${ }^{3}$, Hironari Isshiki ${ }^{1,4}$, Mingwei Chen ${ }^{3}$, Masahiro Yamashita ${ }^{4}$, and \\ Tadahiro Komeda ${ }^{1,5}(\bowtie)$ \\ ${ }^{1}$ Institute of Multidisciplinary Research for Advanced Materials, Tohoku University, Sendai 980-0877, Japan \\ ${ }^{2}$ Center for Nanochemistry, Academy for Advanced Interdisciplinary Studies, Department of Advanced Materials and Nanotechnology, \\ College of Engineering, Beijing University, Beijing 100871, China \\ ${ }^{3}$ WPI-Advanced Institute for Materials Research, Tohoku University, Sendai 980-8577, Japan \\ ${ }^{4}$ Department of Chemistry, Graduate School of Science, Tohoku University, Sendai 980-8578, Japan \\ ${ }^{5}$ CREST, Japan Science and Technology Agency (JST), Saitama 332-0012, Japan \\ Received: 11 May 2010 / Revised: 30 June 2010 / Accepted: 6 July 2010 \\ (C) The Author(s) 2010. This article is published with open access at Springerlink.com
}

\begin{abstract}
Scanning tunneling microscopy/spectroscopy (STM/STS) at $4.8 \mathrm{~K}$ has been used to examine the growth of a double-decker bis(phthalocyaninato)yttrium $\left(\mathrm{YPc}_{2}\right)$ molecule on a reconstructed $\mathrm{Au}(111)$ substrate. Local differential conductance spectra $(\mathrm{d} I / \mathrm{d} V)$ of a single $\mathrm{YPc}_{2}$ molecule allow the characteristics of the highest occupied molecular orbital (HOMO) and the lowest unoccupied molecular orbital (LUMO) to be identified. Furthermore, lateral distributions of the local density of states (LDOS) have also been obtained by $\mathrm{d} I / \mathrm{d} V$ mapping and confirmed by first principles simulations. These electronic feature mappings and theoretical calculations provide a basis for understanding the unique STM morphology of $\mathrm{YP}_{2}$, which is usually imaged as an eight-lobed structure. In addition, we demonstrate that bias-dependent STM morphologies and simultaneous $\mathrm{d} I / \mathrm{d} V$ maps can provide a way of understanding the stability of two-dimensional $\mathrm{YPc}_{2}$ films.
\end{abstract}

\section{KEYWORDS}

Scanning tunneling microscopy/spectroscopy (STM/STS), phthalocyanine, electronic structure

\section{Introduction}

Phthalocyanine $(\mathrm{Pc})$ and its derivatives are very important organic materials, due to their wide applications in areas such as gas sensing devices, photovoltaic materials, and light-emitting diodes [1-3]. The development of scanning tunneling microscopy/spectroscopy (STM/STS) allows a full characterization of the surface assembly and electronic structure of such species on a molecular scale. The first STM observation in this field involved adsorption of $\mathrm{CuPc}$ on $\mathrm{Cu}(100)$, and was followed by exploration of the surface assembly of different $\mathrm{MPc}(\mathrm{M}=\mathrm{Cu}, \mathrm{Co}$, $\mathrm{Fe}, \mathrm{Pb}, \mathrm{Pd}$, and $\mathrm{Mn}$ ) molecules on a variety of metal

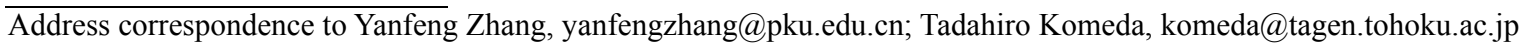


substrates [4-16]. Later on, the characterization of the electronic structure of these molecules became an important topic because molecular orbitals can be detected directly by STS. The resulting electronic structure information can be utilized to explain the complicated STM morphology, and the moleculemolecule and molecule-substrate interactions [17-19]. Exploring the Kondo effects in magnetic ion-containing Pc complexes is another very important research aspect, because of its fundamental significance in solid state physics $[15,16]$.

To date, however, there have been few reported studies by STM under ultrahigh vacuum conditions (UHV) of the assembly behavior or the electronic structure of double-decker or triple-decker metal-Pc complexes on solid substrates. The main reason for this is the thermal instability of these materials during the evaporation process [20-22]. Recently, a single molecule magnet (SMM) of $\mathrm{TbPc}_{2}$ was successfully prepared on $\mathrm{Cu}(111)$, and its spin density was investigated by STM/STS [23]. Further efforts are needed to explore the molecular orbitals of such species and their relationship with the STM morphology, the chemical bonding within the molecule and the novel physical properties of the materials.

In this work, we use a double-decker $\mathrm{YPc}_{2}$ species, because of its good stability during thermal evaporation under UHV. Its growth behavior on $\mathrm{Au}(111)$ has already been reported elsewhere [24]. Here, we try to explore the electronic structure of a single molecule and the stability of $\mathrm{YPc}_{2}$ films. A $4.8 \mathrm{~K} \mathrm{STM} / \mathrm{STS}$ system was used to obtain good STM images, as well as details of the electronic structure. Lateral distributions of the local density of states (LDOS) of a single molecule and of a two-dimensional (2-D) film were achieved using local differential conductance spectra $(\mathrm{d} I / \mathrm{d} V)$ mapping methods. Furthermore, first principles simulations were also carried out in an attempt to explain our experimental observations.

\section{Experimental setup and theoretical calculations}

All the experiments were carried out with a Unisoku low temperature STM composed of an STM chamber and a separate preparation chamber. The base pressure in each chamber was better than $4 \times 10^{-10}$ mbar. The $\mathrm{Au}(111)$ substrate was processed by a standard method of $\mathrm{Ar}^{+}$sputtering followed by annealing. The target molecule was evaporated onto $\mathrm{Au}(111)$ under a flux rate of $\sim 0.1$ monolayer (ML) per minute. After deposition, the sample was instantly transferred to the STM stage which was cooled with liquid helium.

The local differential conductance $(\mathrm{d} I / \mathrm{d} V)$ spectra were recorded by first disabling the feedback loop and then recording the output of a lock-in system. A modulation signal of $4 \mathrm{mV} \mathrm{rms}, 497 \mathrm{~Hz}$ was selected under a tunneling condition of $0.4 \mathrm{~V}, 0.4 \mathrm{nA}$. For $\mathrm{d} I / \mathrm{d} V$ mapping, the feedback loop was kept working while a similar modulation signal to that for the point spectroscopy measurements was applied.

First principles simulations were performed to provide a qualitative illustration of the electronic structure of surface-supported $\mathrm{YPc}_{2}$ molecules. In our simulations, the influence of the Au substrate was not included. The calculations were performed using the projector augmented wave (PAW) method [25] and the generalized gradient-corrected approximation of Perdew, Burke, and Ernzhernof for the exchangecorrelation functional as implemented in the Vienna $a b$ initio simulation package [26,27]. The spinnonpolarized method was employed and $Y 4 s$ and $4 p$ semicore states were treated as valence states to ensure high accuracy. A plane-wave cutoff of $500 \mathrm{eV}$ was used in the calculations and the atomic structure was relaxed until the maximum residual force was less than $10 \mathrm{meV} / \mathrm{A}$. The convergence accuracy of total energy was chosen as $10^{-5} \mathrm{eV}$ in the relaxation of electronic degrees of freedom.

\section{Results and discussion}

The insert in Fig. 1(a) shows the structure of $\mathrm{YPc}_{2}$, which is composed of a $\mathrm{Y}$ atom which is sandwiched by two Pc ligands rotated by $\sim 45^{\circ}$ with respect to each other. Therefore, the $\mathrm{Y}$ atom has an eight-fold coordination with ligand N atoms. Systematic STM examinations were carried out in order to reveal the surface assembly behavior of $\mathrm{YPc}_{2} / \mathrm{Au}(111)$. The typical images in Fig. 1 show that individual molecules appear at low coverage (Fig. 1(a)) and 2-D islands develop at high coverage (Fig. 1(b)) [24]. In addition, square 


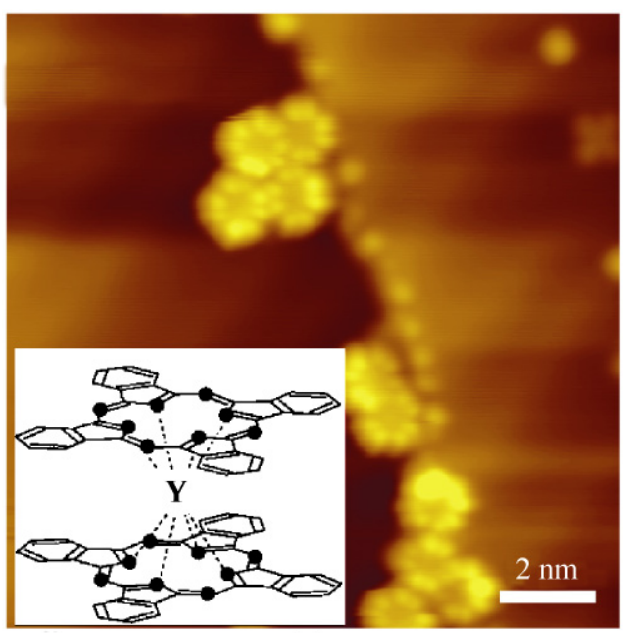

(a)

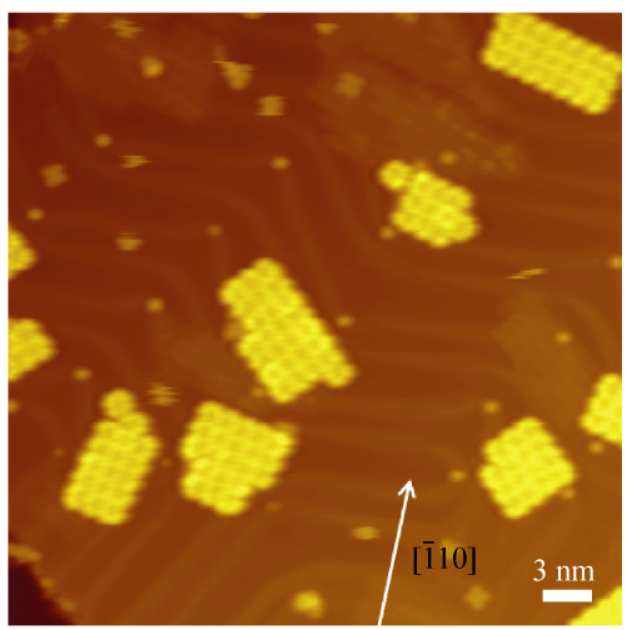

(b)

Figure 1 STM morphologies of $\mathrm{YPc}_{2} / \mathrm{Au}(111)$, characterized for individual molecules at a low molecule density (a) or two-dimensional islands at a high molecule density (b). The molecular structure of $\mathrm{YPc}_{2}$ is shown in the insert in (a)

islands usually dominate on the elbow sites of the herringbone reconstructions of $\mathrm{Au}$, and these sites have been shown to be the nucleation sites of metal atoms and organic molecules.

In order to investigate the system in more detail, we tried to capture STM images of a single molecule. This can only be achieved at low temperature, where the mobilities of molecules decrease and individual molecules can exist. The close-view image of a single molecule (Fig. 2(a)) shows an eight-lobed contrast in the outer part and a weak flat platform in the center of the molecule. This morphology is very different from that of a single-decker Pc adsorbed on Au which is usually observed as a four-lobe shape. We know that the upper and the lower $\mathrm{Pc}$ groups of $\mathrm{YPc}_{2}$ are in different planes and may not be seen by STM with the same contrast. Judging from the line profile of a single molecule (Fig. 2(b)), we get a molecule height of $0.45 \mathrm{~nm}$, more than three times that of a single-decker Pc complex $(0.14 \mathrm{~nm})$. This confirms the presence of $\mathrm{YPc}_{2}$. Therefore the observation of the unique contrast of eight-lobes can be attributed to the overlay of the upper Pc ligand.

In order to study the electronic states of the doubledecker molecule, $\mathrm{d} I / \mathrm{d} V$ spectra (Fig. 2(c), red line) were captured with the STM tip positioned on one of the eight lobes. As a comparison, the spectra of $\mathrm{Au}$ were also measured with the same tip (Fig. 2(c), black line). In this way, we can exclude the possibility of any contribution from the Au substrate. Two sharp peaks appearing at $\pm 1.0 \mathrm{~V}$ can be attributed to the molecular orbitals. Most previous STM/STS studies of single-decker metal-Pc complexes on metal surfaces have reported similar LUMO and HOMO states at $\pm(0.9-1.0 \mathrm{~V})$ [8]. For $\mathrm{YPc}_{2} / \mathrm{Au}(111)$, we can consider the two sharp peaks to arise from the LUMO and HOMO states of the upper Pc ligand. To confirm this, we did first principles calculations and the results are shown in Fig. 2(d). We obtained high densities of electronic states of $\mathrm{C}$ and $\mathrm{N}$ atoms with $V_{\text {bias }}>1.0 \mathrm{~V}$ and $V_{\text {bias }}<-1.0 \mathrm{~V}$. The slight discrepancy between Figs. 2(c) and 2(d) can be attributed to the influence of the $\mathrm{Au}$ substrate not being included in our simple theoretical model. In addition, there is no significant density of electronic states of $Y$ within our focused energy area. To sum up, the molecular orbitals of the Pc ligands make major contributions to the electronic feature of $\mathrm{YPc}_{2}$.

As a rule, the lateral distributions of LDOS can be achieved by performing $\mathrm{d} I / \mathrm{d} V$ mapping measurements, which provide visual images of the distributions of molecular orbitals, and further contribute to identification of the molecule. Figure 3 displays the STM morphology (Fig. 3(a)), and $\mathrm{d} I / \mathrm{d} V$ maps (Figs. 3(b) and $3(\mathrm{c})$ ) of a single molecule at biases of $-0.8 \mathrm{~V}$, and $0.4 \mathrm{~V}$, respectively. The former shows a reasonable 


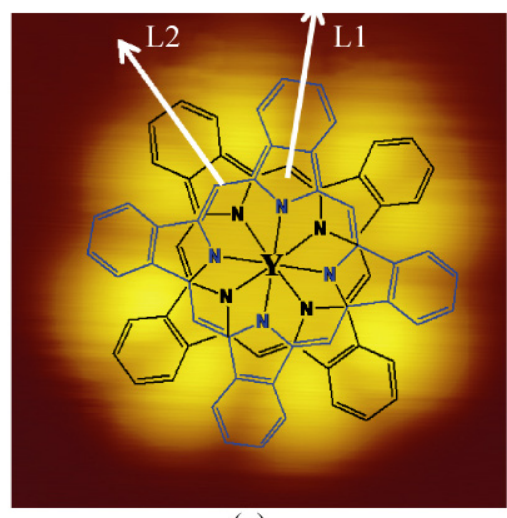

(a)

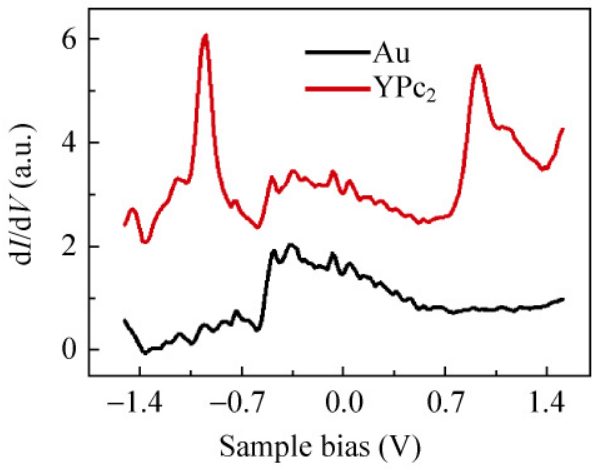

(c)

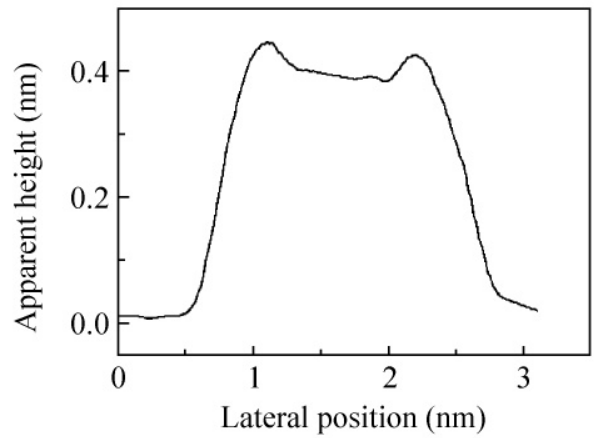

(b)

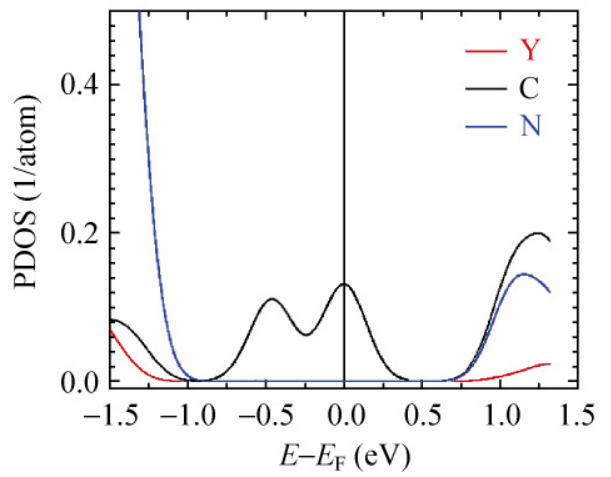

(d)

Figure 2 STM morphology of a $\mathrm{YPc}_{2}$ molecule (a) and its section view along the direction normal to the lobes (b). The inserted arrows indicate the orientations of upper (along L2) and lower (along L1) Pc ligands. (c) $\mathrm{d} I / \mathrm{d} V$ spectra acquired on Pc lobes (red) and on bare $\mathrm{Au}$ (black), under the tunneling condition $V_{\mathrm{B}}=0.4 \mathrm{~V}, I_{\mathrm{T}}=0.4 \mathrm{nA}$, with AC modulation voltage of $4 \mathrm{mV}$ and frequency of $487 \mathrm{~Hz}$. (d) Spinpolarized density of states (PDOS) calculated on Y (red), C (black), and $\mathrm{N}$ (blue) atoms of $\mathrm{YPc}_{2}$

eight-lobed feature. This feature corresponds well with the $\pi$-molecular orbital of the upper Pc ligand if we consider the relative rotation of upper and lower Pc groups. On increasing the bias voltage to $0 \mathrm{~V}$, the occupied molecular orbitals attenuate along the crossing directions of Pc ligands, which is not shown here.

A similar result was found for the unoccupied molecular orbitals, as seen in the mapping data shown in Fig. 3(c). At an intermediate bias of $0.4 \mathrm{~V}$ (Fig. 3(c)), the inner parts of the eight lobes present darker contrasts, and thus two sets of crossed features (without nodes) rotated by $45^{\circ}$ become highlighted. Interestingly, a flat platform appears around the node of the crossings observed by mapping at $0.4 \mathrm{~V}$ (or other voltages below $1 \mathrm{~V}$ ), which may reflect the absence of extra electronic states from the central $\mathrm{Y}$ atom. This can also be deduced from the electronic structure calculations shown in Fig. 2(d), where no significant contribution to the density of states by $\mathrm{Y}$ was observed with bias voltages within the range $\pm 1 \mathrm{~V}$. Our results differ from those for $\mathrm{TbPc}_{2} / \mathrm{Cu}(111)$, where a bright node appears under a similar mapping condition [23]. The brighter/weaker nature of the center, which depends on the electronic states of the metal atoms, may serve as a tracer for recognizing such molecules like $\mathrm{TbPc}_{2}$ and $\mathrm{YPc}_{2}$. It is worth noting that a similar method has been utilized in the identification of CoPc and $\mathrm{CuPc}[6]$.

When mapping above $1.0 \mathrm{~V}$, the periphery of the eight lobes is again highlighted with the formation of an eight-lobed feature (similar to the HOMO states, and therefore not shown here). This variation in $\mathrm{d} I / \mathrm{d} V$ mapping as a function of bias manifests some similarities with the $\mathrm{TbPc}_{2} / \mathrm{Cu}(111)$ system, where theoretical calculations accounted for the eight-lobed HOMO and LUMO d I/d $V$ images [23].

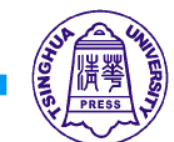




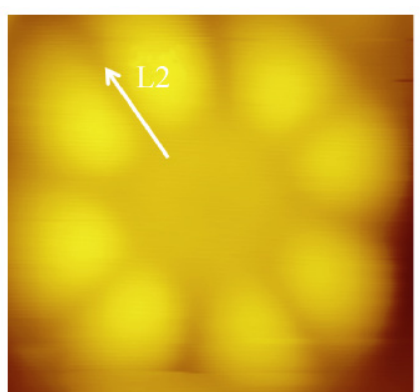

(a)

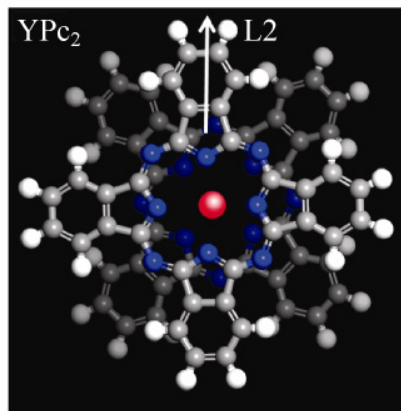

(d)

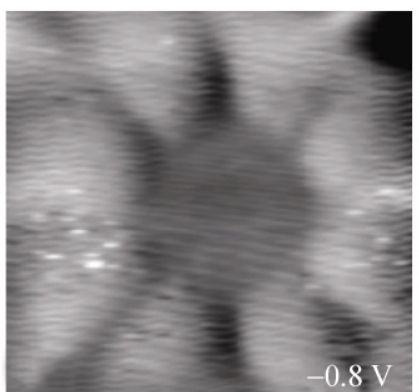

(b)

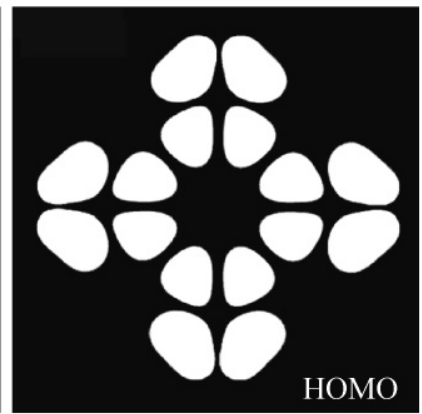

(e)

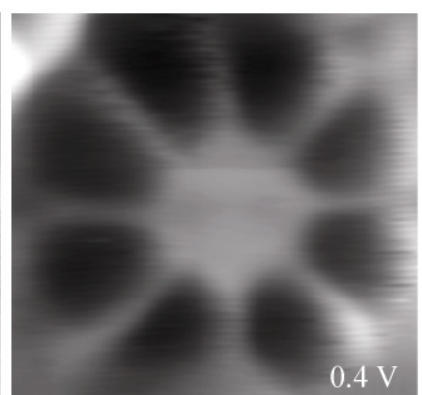

(c)

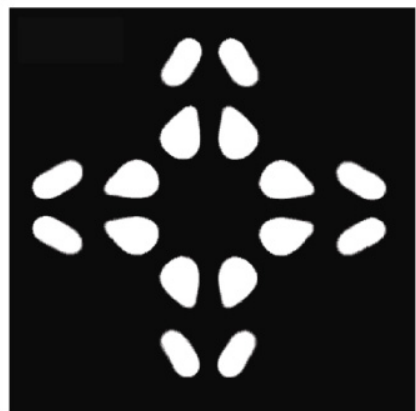

(f)

Figure 3 (a) STM image of a YPc 2 molecule. (b), (c) Conductance maps recorded at biases of (b) $-0.8 \mathrm{~V}$ and (c) $0.4 \mathrm{~V}$. (d) Molecular model of $\mathrm{YPc}_{2}$. (e), (f) Energy-resolved charge densities for (e) the HOMO and (f) an intermediate occupied state deduced by first principles calculations. The arrows in (a) and (d) show the orientations of the upper Pc ligands

To verify our data, we simulated the electronic states of $\mathrm{YPc}_{2}$ by first principles calculations. The structure of $\mathrm{YPc}_{2}$, the HOMO maps and intermediate energy orbital maps (corresponding to a bias voltage lower than the HOMO) are displayed in Figs. 3(d), 3(e), and 3(f), respectively. The HOMO map exhibits four pairs of lobes well delocalized over the $\pi$-molecular orbitals of the upper Pc ligand. With increasing bias voltage, the occupied molecular orbital of Pc shrinks along the direction normal to the benzene rings of the ligand (Fig. 3(f)). As there are no specific electronic states involving $\mathrm{Y}$ and $\mathrm{N}$, no special contrast can be seen along the $\mathrm{Y}-\mathrm{N}$ binding directions. This result suggests that the electronic structure of the upper Pc group should contribute to the eight-lobed STM contrast of $\mathrm{YPc}_{2}$.

Another interesting phenomenon is that our biasdependent STM morphologies usually exhibit complicated features which can not be solely understood by STM imaging. Both $\mathrm{d} I / \mathrm{d} V$ maps and electronic structure characterization may provide a way to solve this problem. Figures 4(a), 4(c), and 4(e) show STM images of a $\mathrm{YPc}_{2}$ film recorded at biases of $-0.8 \mathrm{~V}$, $-0.4 \mathrm{~V}$, and $0.4 \mathrm{~V}$, respectively. Most of the molecules manifest an eight-lobed shape on imaging at $-0.8 \mathrm{~V}$ (Fig. 4(a)). A few molecules with an eight-lobed shape show higher contrast than neighboring ones on scanning at $-0.4 \mathrm{~V}$ (Fig. 4(c)). Darker molecules with a four-lobed shape appear on scanning at $0.4 \mathrm{~V}$ (Fig. 4(e), indicated by dashed circles). Fuzzy spots on the film surface (Figs. 4(c) and 4(e)) may be due to the instability of the upper Pc ligands during the scanning process; it cannot be a tip effect since the STM tip always remains in good condition.

Simultaneous $\mathrm{d} I / \mathrm{d} V$ maps are displayed in the right column of Fig. 4 . For $\mathrm{d} I / \mathrm{d} V$ mapping at $-0.8 \mathrm{~V}$, most of the molecules exhibit an eight-lobed shape (Fig. 4(b)). This is comparable to the HOMO state of a single molecule (Figs. $3(\mathrm{~b})$ and $3(\mathrm{e})$ ). At $\pm 0.4 \mathrm{~V}$, a majority of the molecules are mapped as two sets of crossings with central flat nodes (Figs. 4(d) and 4(f)). Again they show a good correspondence with the single molecule mapping data (Fig. 3(c)). In addition, we can also identify some abnormal molecules in the 


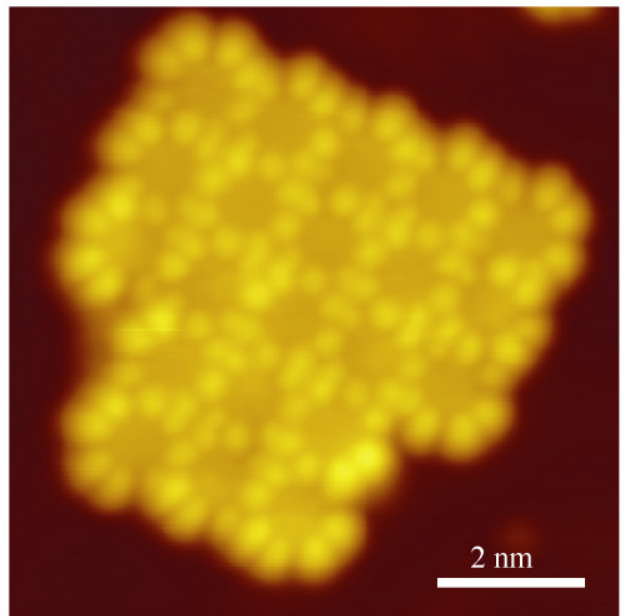

(a)

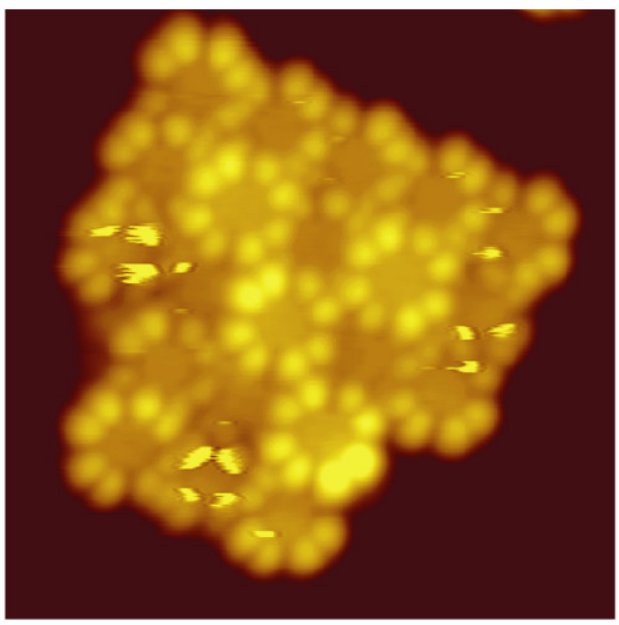

(c)

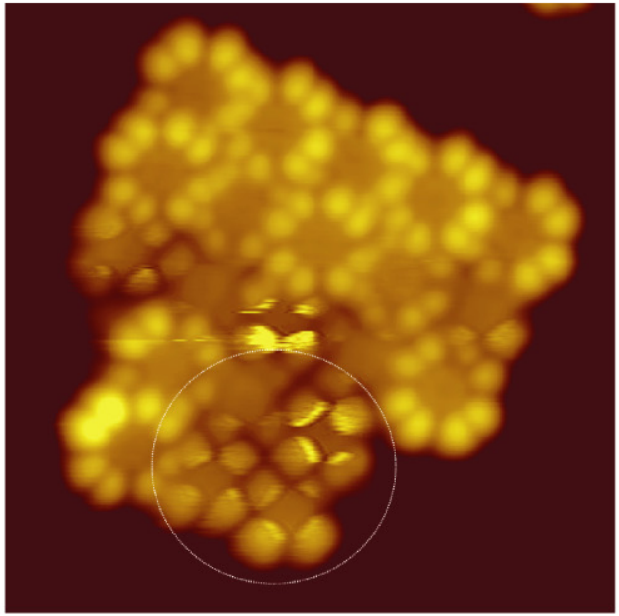

(e)

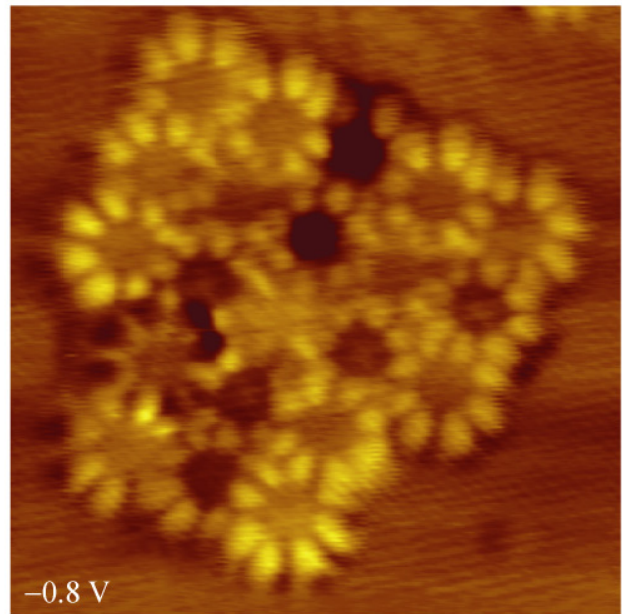

(b)

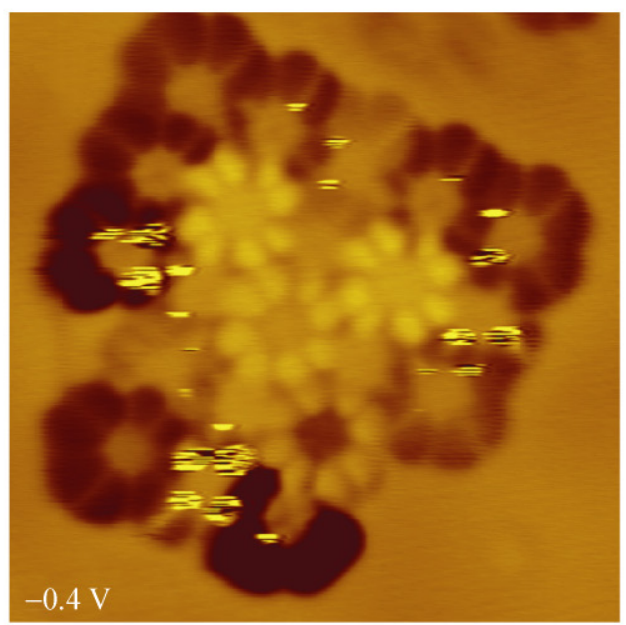

(d)

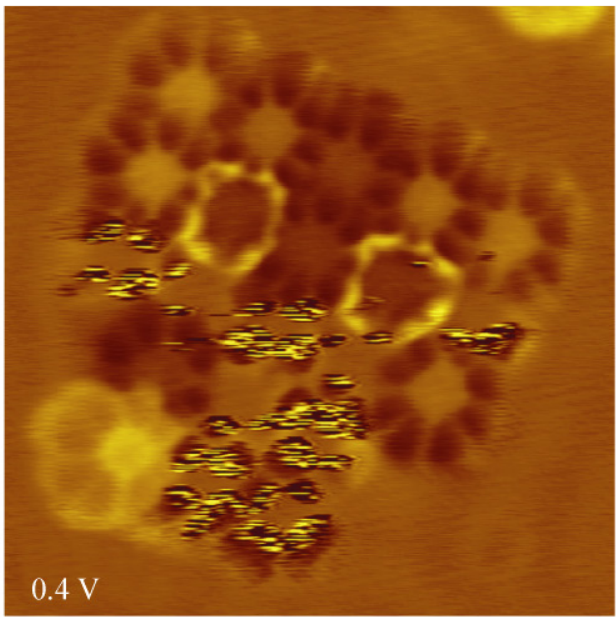

(f)

Figure 4 (a), (c), (e) STM morphologies of a $\mathrm{YPc}_{2}$ island recorded at biases of (a) $-0.8 \mathrm{~V}$, (c) $-0.4 \mathrm{~V}$, and (e) $0.4 \mathrm{~V}$. The corresponding $\mathrm{d} I / \mathrm{d} V$ maps are shown in (b), (d), and (f) respectively 
middle part of the film, which present an eight-lobed feature (Figs. 4(d) and 4(f)). These abnormal HOMO/ LUMO images may indicate that these molecules are located in a different chemical environment from the neighboring ones [28].

In Fig. 4(e), some molecules are imaged as darker contrasts of four-lobed shapes, and their apparent height approaches $0.25 \mathrm{~nm}$. This phenomenon emerges only after repetitive scanning of a 2-D film for a long time (from Fig. 4(a) to Fig. 4(c)). The mechanism of this morphology evolution is not clear since the film is kept at $4.8 \mathrm{~K}$ where thermal instability is essentially eliminated. These shape alternations for $\mathrm{YPc}_{2}$ may result from desorption of the upper Pc groups. In this case, the probable driving force is the applied bias that will generate an extra electric field between tip and sample. In the $\mathrm{d} I / \mathrm{d} V$ mapping images, we can identify corresponding evolutions of the electronic states around these four-lobed molecules (Figs. 4(d) and 4(f)). Based on these tentative results, we can conclude that double-decker $\mathrm{YPc}_{2}$ films may not be stable throughout the STM imaging process. Since along the direction normal to the surface, the upper Pc groups are only coordinated with the central $\mathrm{Y}$ atom and kept apart from the substrate, this will induce weak interactions between the upper Pc group and the Au substrate. The external field induced by tip bias may therefore result in desorption of the upper Pc ligands. This is only a preliminary hypothesis and more experimental data are needed, such as measuring an I-t curve to see whether there is a current drop when the desorption occurs.

\section{Conclusions}

A STM/STS system at $4.8 \mathrm{~K}$ and first principles calculations have been utilized to explore the electronic structure of $\mathrm{YPc}_{2}$ prepared on $\mathrm{Au}(111)$. Similar to other double-decker metal-Pc complexes, HOMO and LUMO $\mathrm{d} I / \mathrm{d} V$ mapping images of $\mathrm{YPc}_{2}$ show eight-lobed features in both experimental results and theoretical calculations. The STM morphology of Y $\mathrm{Pc}_{2}$ involving an eight-lobed shape can be explained by the influence of the electronic structure of the upper Pc group. In addition, based on our STM/STS results for 2-D films, the change in morphology of $\mathrm{YPc}_{2}$ from eight-lobed to four-lobed shapes may be understood to arise from the desorption of the upper Pc ligands. Therefore our experiments suggests that, even under STM observations at $4.8 \mathrm{~K}$, double-decker metal-Pc complexes have a limited stability.

\section{Acknowledgements}

The first author acknowledges the financial support of JSPS (Japan Society for the Promotion of Science). This work was also supported by an International Collaborative Research Grant by the National Institute of Information and Communications Technology of Japan. We also would like to thank the Center for Computational Materials Science, Institute for Materials Research, Tohoku University for supporting the use of the Hitachi SR11000 (model K2) supercomputing system.

Open Access: This article is distributed under the terms of the Creative Commons Attribution Noncommercial License which permits any noncommercial use, distribution, and reproduction in any medium, provided the original author(s) and source are credited.

\section{References}

[1] Joachim, C.; Gimzewski, J. K.; Aviram, A. Electronics using hybrid-molecular and mono-molecular devices. Nature 2000, 408, 541-548.

[2] Cracium, M. F.; Rogge, S.; Morpurgo, A. F. Correlation between molecular orbitals and doping dependence of the electrical conductivity in electron-doped metal-phthalocyanine compounds. J. Am. Chem. Soc. 2005, 127, 12210-12211.

[3] Papageorgiou, N.; Salomon, E.; Angot, T.; Layet, J. M.; Giovanelli, L.; Lay, G. L. Physics of ultra-thin phthalocyanine films on semiconductors. Prog. Surf. Sci. 2004, 77, 139-170.

[4] Lippel, P. H.; Wilson, R. J.; Miller, M. D.; Woll, Ch.; Chiang, S. High-resolution imaging of copper-phthalocyanine by scanning-tunneling microscopy. Phys. Rev. Lett. 1989, 62, 171-174.

[5] Hipps, K. W.; Lu, X.; Wang, X. D.; Mazur, U. Metal d-orbital occupation-dependent images in the scanning tunneling microscopy of metal phthalocyanines. J. Phys. Chem. 1996, 100, 11207-11210.

[6] Lu, X.; Hipps, K. W.; Wang, X. D.; Mazur, U. Scanning tunneling microscopy of metal phthalocyanines: $\mathrm{d}^{7}$ and $\mathrm{d}^{9}$ cases. J. Am. Chem. Soc. 1996, 118, 7197-7202. 
[7] Chizhov, I.; Scoles, G.; Kahn, A. The influence of steps on the orientation of copper phthalocyanine monolayers on $\mathrm{Au}(111)$. Langmuir 2000, 16, 4358-4361.

[8] Takada, M.; Tada, H. Scanning tunneling microscopy and spectroscopy of phthalocyanine molecules on metal surfaces. Jpn. J. Appl. Phys. 2005, 44, 5332-5335.

[9] Chen, L.; Hu, Z. P.; Zhao, A. D.; Wang, B.; Luo, Y.; Yang, J. L.; Hou, J. G. Mechanism for negative differential resistance inmolecular electronic devices: Local orbital symmetry matching. Phys. Rev. Lett. 2007, 99, 146803.

[10] Zhao, A. D.; Li, Q. X.; Chen, L.; Xiang, H. J.; Wang, W. H.; Pan, S. A.; Wang, B.; Xiao, X. D.; Yang, J. L.; Hou, J. G.; Zhu, Q. S. Controlling the Kondo effect of an adsorbed magnetic ion through its chemical bonding. Science 2005, 309, 1542-1544.

[11] Jiang, P.; Ma, X. C.; Ning, Y. X.; Song, C. L.; Chen, X.; Jia, J. F., Xue, Q. K. Quantum size effect directed selective self-assembling of cobalt phthalocyanine on $\mathrm{Pb}(111)$ thin films. J. Am. Chem. Soc. 2008, 130, 7790-7791.

[12] Cheng, Z. H.; Gao, L.; Deng, Z. T.; Jiang, N.; Liu, Q.; Shi, D. X.; Du, S. X.; Guo, H. M.; Gao, H. J. Adsorption behavior of iron phthalocyanine on $\mathrm{Au}(111)$ surface at submonolayer coverage. J. Phys. Chem. C 2007, 111, 9240-9244.

[13] Strohmaier, R.; Ludwig, C.; Petersen, J.; Gompf, B.; Eisenmenger, W. Scanning tunneling microscope investigations of lead-phthalocyanine on $\mathrm{MoS}_{2}$. J. Vac. Sci. Technol. B 1996, 14, 1079-1082.

[14] Gopakumar, T. G.; Lackinger, M.; Hackert, M.; Muller, F.; Hietschold, M. Adsorption of palladium phthalocyanine on graphite: STM and LEED study. J. Phys. Chem. B 2004, 108, 7839-7843.

[15] Fu, Y. S.; Ji, S. H.; Chen, X.; Ma, X. C.; Wu, R.; Wang, C. C.; Duan, W. H.; Qiu, X. H.; Sun, B.; Zhang, P.; Jia, J. F.; Xue, Q. K. Manipulating the Kondo resonance through 1uantum size effects. Phys. Rev. Lett. 2007, 99, 256601.

[16] Gao, L.; Ji, W.; Hu, Y. B.; Cheng, Z. H.; Deng, Z. T.; Liu, Q.; Jiang, N.; Lin, X.; Guo, W.; Du, S. X.; Hofer, W. A.; Xie, X. C.; Gao, H. J. Site-specific Kondo effect at ambient temperatures in iron-based molecules. Phys. Rev. Lett. 2007, 99, 106402.

[17] Nazin, G. V.; Qiu, X. H.; Ho, W. Visualization and spectroscopy of a metal-molecule-metal bridge. Science 2003, 302, 77-81.

[18] Qiu, X. H.; Nazin, G. V.; Ho, W. Vibronic states in single molecule electron transport. Phys. Rev. Lett. 2004, 92, 206102.
[19] Nilius, N.; Simic-Milosevic, V. Adsorption of single magnesium phthalocyanine molecules on $\mathrm{V}_{2} \mathrm{O}_{3}$ thin films. $J$. Phys. Chem. C 2008, 112, 10027-10031.

[20] Yang, Z. Y.; Gan, L. H.; Lei, S. B.; Wan, L. J.; Wang, C.; Jiang, J. Z. Self-assembly of PcOC8 and its sandwich lanthanide complex $\operatorname{Pr}(\mathrm{PcOC} 8)_{2}$ with oligo(phenylene-ethynylene) molecules. J. Phys. Chem. B 2005, 109, 19859-19865.

[21] Takami, T.; Arnold, D. P.; Fuchs, A. V.; Will, G. D.; Goh, R.; Waclawik, E. R.; Bell, J. M.; Weiss, P. S.; Sugiura, K.; Liu, W.; Jiang, J. Two-dimensional crystal growth and stacking of bis(phthalocyaninato) rare earth sandwich complexes at the 1-phenyloctane/graphite interface. J. Phys. Chem B. 2006, 110, 1661-1664.

[22] Lei, S. B.; Deng, K.; Yang, Y. L.; Zeng, Q. D.; Wang, C.; Jiang, J. Z. Electric driven molecular switching of asymmetric tris(phthalocyaninato) lutetium triple-decker complex at theliquid/solid interface. Nano. Lett. 2008, 8, 1836-1843.

[23] Vitali, L.; Fabris, S.; Conte, A. M.; Brink, S.; Ruben, M.; Baroni, S.; Kern, K. Electronic structure of surfacesupported bis(phthalocyaninato) terbium(III) single molecular magnets. Nano. Lett. 2008, 8, 3364-3368.

[24] Zhang, Y, F.; Isshiki, H.; Katoh, K.; Yoshida, Y.; Yamashita, M.; Miyasaka, H.; Breedlove, B. K.; Kajiwara, T.; Takaishi, S.; Komeda, T. Low-temperature scanning tunneling microscopy investigation of bis(phthalocyaninato)yttrium growth on $\mathrm{Au}(111)$ : From individual molecules to two-dimensional domains. J. Phys. Chem. C 2009, 113, 9826-9830.

[25] Kresse, G.; Joubert, D. From ultrasoft pseudopotentials to the projector augmented-wave method. Phys. Rev. B 1999, 59, 1758-1775.

[26] Perdew, J. P.; Burke, K.; Ernzerhof, M. Generalized gradient approximation made simple. Phys. Rev. Lett. 1996, 77, 3865-3868.

[27] Kresse, G.; Furthmuller, J. Efficiency of ab-initio total energy calculations for metals and semiconductors using a planewave basis set. Comput. Mater. Sci. 1996, 6, 15-50.

[28] Katoh, K.; Yoshida, Y.; Yamashita, M.; Miyasaka, H.; Breedlove, B. K.; Kajiwara, T.; Takaishi, S.; Ishikawa, N.; Isshiki, H.; Zhang, Y. F.; Komeda, T.; Yamagishi, M.; Takeya, J. Direct observation of lanthanide( III)-phthalocyanine molecules on $\mathrm{Au}(111)$ by using scanning tunneling microscopy and scanning tunneling spectroscopy and thin-film field-effect transistor properties of $\mathrm{Tb}(\mathrm{III})$ - and Dy( III)-phthalocyanine molecules. J. Am. Chem. Soc. 2009, 131, 9967-9976. 